TP Periodica Polytechnica Electrical Engineering and Computer Science

60(1), pp. 82-87, 2016

DOI: $10.3311 /$ PPee.9043

Creative Commons Attribution (i)

RESEARCH ARTICLE

\section{Stability Problems of High Frequency Signals Generated by Direct Mixing Two Laser Beams}

\author{
Gábor Fekete $^{1 *}$, Gergely Mészáros ${ }^{1}$, Tibor Berceli ${ }^{1}$, Eszter Udvary ${ }^{1}$
}

Received 17 November 2015; accepted 12 January 2016

\begin{abstract}
In this paper we study the radio frequency $(R F)$ signal properties generated by direct mixing two laser beams, which can be used in very high frequency (e.g. terahertz) communications. We show the supply source stability has a critical effect in this procedure. It can broaden the RF signal spectrum up to $1 \mathrm{GHz}$. Although the linewidth of the lasers is the main factor determining the spectral width of the generated RF signal, the supply source instability also has a very significant contribution. In this paper we show the effect of these parameters by simulations and measurements.
\end{abstract}

\section{Keywords}

photomixing, direct laser mixing, optically generated radio frequency signal, carrier stability, laser linewidth, power supply stability

${ }^{1}$ Department of Broadband Infocommunications and Electromagnetic Theory, Faculty of Electrical Engineering and Informatics,

Budapest University of Technology and Economics,

H-1111 Budapest, Egry József utca 18, Hungary

*Corresponding author, e-mail: gfekete@hvt.bme.hu

\section{Introduction}

The radio frequency (RF) information transmission by radiation is widely used because of the easy installation of these links. However, the frequency bands allocated for communications are limited and they are already saturated because of the popularity of mobile networks. High data rate communications usually has to cover small areas (tens of $\mathrm{km}$ ), because it is used frequently to transmit the raw video from the stadium to the TV studio for further signal processing. These kinds of applications are possible by using $100 \mathrm{GHz}$ and higher frequencies as carriers. The conventional RF techniques at this frequency cannot be used or it is too expensive for commercial application. At the receiver side the uni-traveling-carrier photodiode (UTCPD) is applied for the terahertz signal detection, which limits the data rate and distance of the transmission. Many papers presented [1-4] a demo terahertz system using these diodes, but the achieved maximum transmission distances were around $1 \mathrm{~m}$. At the transmitter side the critical point is the carrier generation. Its frequency has to be stable with small phase noise.

Recently the carrier generation in the terahertz frequency domain is done more frequently by optical processes, e.g. mixing two laser beams. A significant benefit of the optical carrier generation is the easy tuneability. It provides fast and wide band tuning. In the optical solution the noise does not depend on the tuning facility.

The aim of the present paper is to investigate the stability problems of high frequency signals generated by direct mixing two laser beams. The investigations are carried out by doing a case study using simulations. The effects of the laser linewidth and the supply source stability are studied on the noise spectral width of the generated RF signal.

\section{Mixing two independent laser beams}

Mixing two independent laser beams is the simplest RF carrier generation method using optical components. It needs only two lasers (at different frequencies), a photodiode and an optical coupler, which couples the two laser beams into a photodiode as shown in Fig. 1. The signals of two continuous wave $(\mathrm{CW})$ lasers are combined by a high directivity coupler 
or hybrid avoiding this way any interactions between the lasers like mutual injection locking by reflections due to mismatches. The mutual injection locking cannot occur in the case when the laser locking bands do not overlap each other. The combined two laser beams are mixed in a photodiode. The photodiode has a quadratic characteristic therefore the frequency difference of the two CW lasers, the radio frequency, will appear at the output of the photodiode beside the DC component from the unmodulated laser sources. The photodiode signal can be written as [7]:

$$
P=K\left|E_{C W 1}+E_{C W 2}\right|^{2}
$$

where $\mathrm{K}$ is a proportionality constant. The signals of the $\mathrm{CW}$ lasers have the following complex forms [7]:

$$
\begin{aligned}
& E_{C W 1}=A_{C W 1} \cdot \exp \left(-j\left[\omega_{1} t+\Theta_{1}\right]\right) \\
& E_{C W 2}=A_{C W 2} \cdot \exp \left(-j\left[\omega_{2} t+\Theta_{2}\right]\right)
\end{aligned}
$$

where $\mathrm{A}$ is the amplitude, $\Theta$ is the phase, and $\omega$ is the angular frequency of the laser sources. Substituting Eq. (2) into Eq. (1), we get the mathematical form of the electrical signals at the output of the photodiode [7]:

$$
\begin{aligned}
P(t) & =K A_{C W 1}^{2}+K A_{C W 2}^{2} \\
& +2 K A_{C W 1} A_{C W 2} \cos \left(\omega_{R F} t+\Theta_{1}+\Theta_{2}\right)
\end{aligned}
$$

where $\omega_{\mathrm{RF}}$ is the frequency difference between the two $\mathrm{CW}$ lasers:

$$
\omega_{R F}=\omega_{1}-\omega_{2}
$$

The benefit of the photodiode signal mixing is the lack of the other mixing product, $\omega_{\mathrm{RF} 2}$.

$$
\omega_{R F 2}=\omega_{1}+\omega_{2}
$$

The photodiodes have limited bandwidth therefore the sum frequency product of photomixing is usually out of the photodiode bandwidth. The RF is determined by the frequencies of the two CW lasers. The laser wavelength is dependent on the bias current and consequently varying the bias current the frequency of the mixing product is changed.

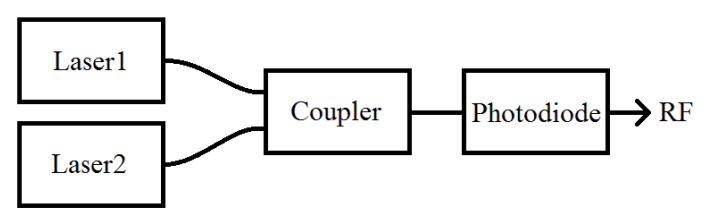

Fig. 1 Direct carrier generation method for terahertz carrier signals.

The generated RF can be tuned by temperature or by using a tuneable laser source. In the former case the tuning frequency range is around a few hundred $\mathrm{GHz}$, which can be enough in most cases. Frequency tuning with temperature is one benefit of that method.
Table 1 Parameters of the simulated and measured laser.

\begin{tabular}{ll}
\hline \multicolumn{2}{c}{ Simulation } \\
\hline Laser nominal frequency & $193.12 \mathrm{THz}$ \\
Relative Intensity Noise & $-130 \mathrm{~dB} / \mathrm{Hz}$ \\
Optical power & $1 \mathrm{~mW}$ \\
Current Injection Efficiency & 1 \\
Current (if not swept) & $100 \mathrm{~mA}$ \\
Linewidth (if not swept) & $20 \mathrm{MHz}$ \\
Wavelength-current coefficient & $0.04 \mathrm{~nm} / \mathrm{mA}$ \\
\hline \multicolumn{1}{c}{ Measurement } & \\
\hline DWDM laser linewidth & $20 \mathrm{MHz}$ \\
DWDM laser current & $20 \mathrm{~mA}$ \\
Laboratory laser linewidth & $50 \mathrm{MHz}$ \\
Laboratory laser power & $3 \mathrm{dBm}$ \\
Wavelength-current coefficient & $0.04 \mathrm{~nm} / \mathrm{mA}$ \\
\hline
\end{tabular}

\section{Simulations}

Simulations and measurements were made at $10 \mathrm{GHz}$, because we would like to demonstrate the effect of the power supply instability and laser linewidth also by measurements and not only by simulations. Lasers from the shelves provide direct mixing methods up to $20 \mathrm{GHz}$, therefore we selected the half of that frequency range. The linewidth and the power supply instability effects are similar at $10 \mathrm{GHz}$ compared to $100 \mathrm{GHz}$ but in the former case they have relatively higher effect on the RF signal, which can help their investigation.

The quality of the generated RF signal is determined by the CW lasers. The linewidth, the temperature and the forward current of the lasers are the main factors which highly affect the RF signal quality. The dependence of the RF signal from these factors, except the temperature, was simulated by VPItransmissionMaker (VPI). The laser temperature is used for tuning the $\mathrm{RF}$ in this method. Because it is a controlled parameter, which directly affects the RF signal, the temperature dependence of the lasers was not simulated. The radio frequency accuracy depends on the temperature control loop stability of the lasers. Fast temperature control results in large overshoot which will tune away the laser frequency. Therefore, the speed of the control loop has to be as small as possible to avoid fast frequency changes caused by the temperature variations. On the other hand, the temperature has to be kept very stable or the RF will be tuned away by $10 \mathrm{GHz}$ or more. It depends on the temperature coefficient of the laser.

The emitted light frequency of the laser changes, when the laser current is increased or decreased. There is always a small change in the power supply, so this uncertainty can appear as the instability of the radio frequency. This effect was simulated 
by the VPI. However, the applied laser diode model was different from our laser. It comes from simulation technique considerations. Not all laser diode models can be used to simulate the power change caused wavelength shift and those models, which simulate this effect, are more complex and the parameters cannot be obtained from the datasheet. On the other hand, showing the current caused wavelength shift was more important, than creating a proper model for our laser diode. In the VPI we used the transmission line based laser model. It provides the most accurate simulation in the VPI (noise, linewidth, ect.). This model was different from our laser because of the previously mentioned reasons. Based on the applied laser diode model the frequency changed by $1 \mathrm{GHz}$ if the bias current was increased or decreased by $1 \mathrm{~mA}$. This can cause high instability in the RF signal. In the simulation the laser current was set to $100 \mathrm{~mA}$. Firstly, one of the current supply stability was set to $1 \%$ tolerance, while the other current source provided constant current. The stability means that the current of the source could change $1 \%$ of the given constant value. In this case it was $1 \mathrm{~mA}$. This change followed a uniform distribution. The effect of the supply instability can be observed on the blue curve in Fig. 2. The frequency of the detected signal was shifted in a $2 \mathrm{GHz}$ wide spectrum range. The RF was set to $10 \mathrm{GHz}$ so this uncertainty causes $20 \%$ changes in the carrier frequency. It can highly distort the signal transmission. Therefore, the power stability is much more important in this technique than we thought it first. Secondly, the power supply stability was changed by $3 \%$. The effect of the higher instability in the supply current can be seen by the red curve in Fig. 2. The frequency shift was increased to threefold, up to $6 \mathrm{GHz}$.

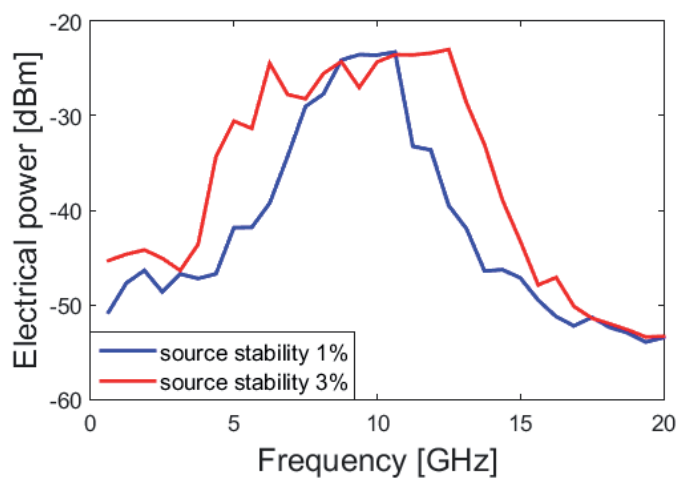

Fig. 2 Current source instability caused shift in the RF frequency.

After that the currents of both lasers were changed by $1 \%$. The result can be seen in the black curve of Fig. 3. This produced $3 \mathrm{GHz}$ frequency uncertainty in the detected signal, which is only $1 \mathrm{GHz}$ higher compared to the case when only one of the current sources was ideal. This simulation showed that both supply affects the frequency of the RF signal. In the worst case it will double the frequency shift compared to the first simulation.

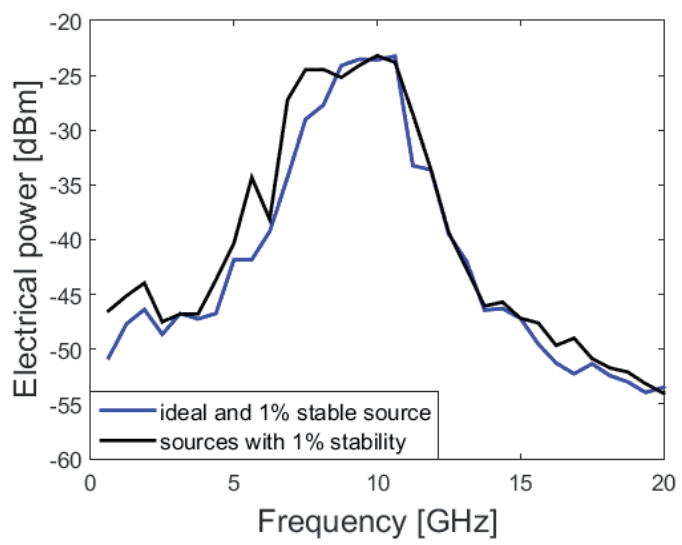

Fig. 3 Black curve: the stability of both laser current sources was $1 \%$; blue curve: one of the laser source was ideal while the current of the other source was changed by $1 \%$.

Higher supply current increases the uncertainty value of the power supply, so the bandwidth of the generated RF will be wider. Therefore, supply current was set to $300 \mathrm{~mA}$, but the stability remained at $1 \%$. The result of this simulation can be seen in Fig. 4 (green curve). The blue one shows the previous case when the supply current was $100 \mathrm{~mA}$. As we expected higher supply current with the same stability caused a larger frequency shift in the detected signal. This instability was $6 \mathrm{GHz}$, which is the same when supply current was $100 \mathrm{~mA}$ with $3 \%$ instability. Although, the high supply current increases the power of the radiated terahertz wave, there are limitations in this respect due to the saturation and the conversion efficiency of the photodiode.

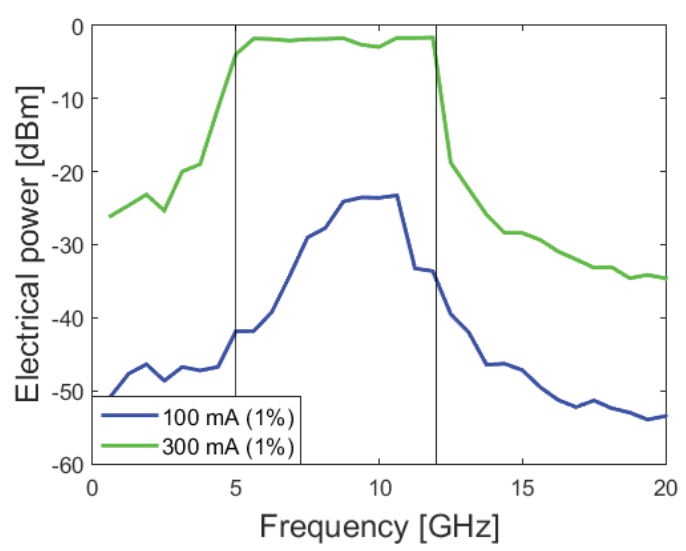

Fig. 4 RF instability if the supply current is $100 \mathrm{~mA}$ (blue) and $300 \mathrm{~mA}$ (green). The stability of the supply is $1 \%$.

The instability in the radio frequency is determined by the less stable power supply. It can be seen in Fig. 5. If the two lasers were supplied by $1 \%$ and $3 \%$ stable current sources (green curve), it gave similar result to that case when the lasers were driven by an ideal current source and a source with $3 \%$ uncertainty (red curve). In both cases the frequency change was 5-6 GHz. 


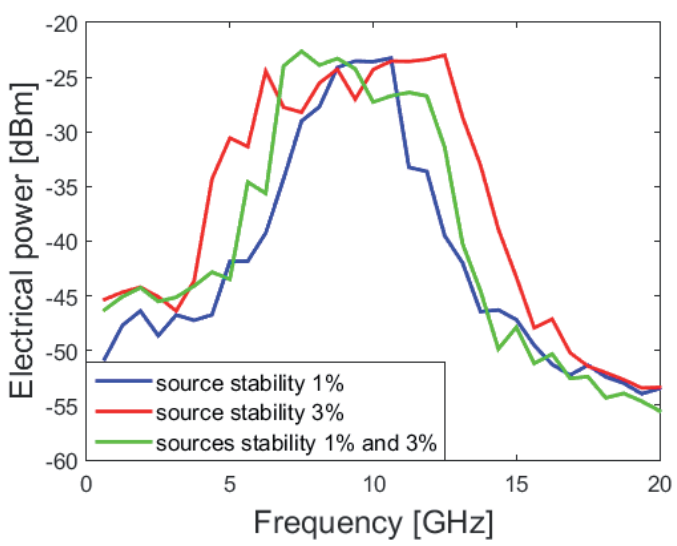

Fig. 5 Power supplies caused frequency instability. Blue: ideal source and a current source with $1 \%$ uncertainty. Red: ideal source and a current source with $3 \%$ uncertainty. Green: current sources with $1 \%$ and $3 \%$ uncertainty.

Our laser linewidth is between $5 \mathrm{MHz}$ and $100 \mathrm{MHz}$, but in the simulation it was set from the ideal (zero linewidth) up to $100 \mathrm{MHz}$. However, the first and last values are important only theoretically. Higher laser linewidth increases the phase noise therefore the RF signal accuracy will be worse. Zero linewidth means the laser emits light only at a single frequency. Increasing the laser linewidth its spectral bandwidth is increased, too. In the simulation the non-zero linewidth modulates the laser frequency. This kind of frequency modulation is driven by a white noise source which has a Gaussian distribution. The variance of it is the linewidth parameter of the laser multiplied by $2 \pi$. This modulation can cause that the RF signal bandwidth is smaller or larger than the lasers linewidth.

Table 2 summarizes the effect of the laser linewidth on the RF signal. In the simulation an ideal photodiode was used because we would like to observe the photomixing effect without any distortion. In the first simulation laser2 was ideal i.e. with zero linewidth and the laser1 linewidth was changed. The bandwidth of RF did not change linearly with the linewidth. Up to few $\mathrm{MHz}$ the detected bandwidth of RF was approximately constant, $70 \mathrm{MHz}$. RF bandwidth starts to increase when the laser linewidth was higher than $100 \mathrm{MHz}$. 20 times higher linewidth doubled the RF signal bandwidth. However, this ratio is not so high, and this kind of uncertainty is not too much in the terahertz range, but it can be critical if a signal with narrow bandwidth is transmitted.

Table 2 contains the case when the other laser linewidth was $20 \mathrm{MHz}$. It was set to $20 \mathrm{MHz}$ because one of our laser linewidth has the same value. The simulated values of RF bandwidth are similar to the previous case, when one of the lasers was ideal. Simulations show that the RF bandwidth is determined by that laser which has larger linewidth. From the simulation results it can be seen that a very small laser linewidth does not decreases the RF signal bandwidth below a bottom value. In our case it was $68 \mathrm{MHz}$ and it increased as the laser had higher linewidth. However, the spectral broadening of the RF signal is a nonlinear function. It was saturated about $100 \mathrm{MHz}$. This dependence between the RF bandwidth and the laser linewidth is not too high and may be another effect covers it, but it exists and we have to take it into consideration. The linewidth and the laser power are not independent from each other. Higher laser power decreases the laser linewidth. The relation between them can be written as [7]:

$$
\Delta f=K \frac{1}{P}
$$

where $\mathrm{K}$ is a proportionality constant, $\mathrm{P}$ is the laser power and $\Delta \mathrm{f}$ is the laser linewidth. The above equation shows that higher laser power will decrease the linewidth of the lasers. However, the laser linewidth has small effect on the RF signal, therefore the high laser power caused other distortion can be larger than the decreased linewidth caused signal improvement.

\begin{tabular}{|c|c|c|}
\hline \multicolumn{2}{|c|}{ Linewidth $[\mathrm{MHz}]$} & \multirow{2}{*}{$\begin{array}{l}\text { RF signal }(3 \mathrm{~dB}) \\
\text { bandwidth }[\mathrm{MHz}]\end{array}$} \\
\hline Laser1 & Laser2 & \\
\hline 0.1 & 0 & 68 \\
\hline 1 & 0 & 70 \\
\hline 20 & 0 & 71 \\
\hline 50 & 0 & 79 \\
\hline 100 & 0 & 89.4 \\
\hline 20 & 0.1 & 72 \\
\hline 20 & 1 & 72 \\
\hline 20 & 20 & 72.4 \\
\hline 20 & 50 & 76 \\
\hline 20 & 100 & 81.2 \\
\hline
\end{tabular}

\section{Measurements}

For the measurements two kinds of lasers were used. One of the lasers was a laboratory laser source with high stability and a linewidth lower than $50 \mathrm{MHz}$. The other one was a commercial laser source. It worked in a DWDM (Dense Wavelength Division Multiplexing) system, before it was dismounted from the board. Its linewidth is $20 \mathrm{MHz}$ and its temperature coefficient is $0.1 \mathrm{~nm} / \mathrm{C}^{\circ}$. The $\mathrm{RF}$ was set up by the temperature to $10 \mathrm{GHz}$. Fig. 6 shows the schematic of the measurements. The opticalelectric converter $(\mathrm{O} / \mathrm{E})$ contains a photodiode and an amplifier with $20 \mathrm{~dB}$ gain.

First, the power supply effect on the RF signal was examined. Three cases were measured: DWDM lasers supplied by a laboratory voltage source (used as a current source), then one of the voltage sources was exchange to a laser driver, and finally both of them were supplied by laser drivers. Each DWDM laser current was $20 \mathrm{~mA}$. The results are summarized in Table 3. 


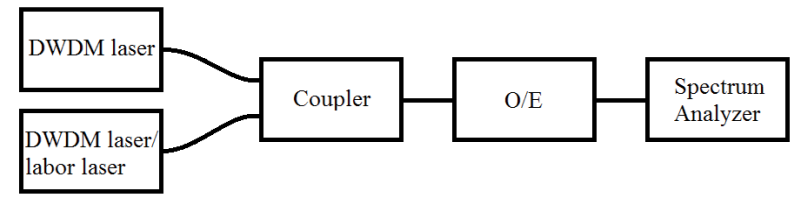

Fig. 6 Schematic of the measurements.

Table 3 Power supply instability caused uncertainty in the RF, if the supply current is $20 \mathrm{~mA}$.

\begin{tabular}{lll}
\hline Photomixed lasers & $\begin{array}{l}\text { Supply source of the } \\
\text { DWDM lasers }\end{array}$ & $\begin{array}{l}\text { RF uncertainty } \\
{[\mathrm{MHz}]}\end{array}$ \\
\hline & voltage sources & 315 \\
two DWDM lasers & voltage source, laser driver & $147 / 135$ \\
& laser drivers & 66 \\
\hline $\begin{array}{l}\text { DWDM laser }- \text { with } \\
\text { a laboratory laser }\end{array}$ & voltage source & 490 \\
\hline
\end{tabular}

Voltage source type power supplies were used for the measurements because in the commercial equipments voltage sources are used or the required current comes from a voltage source. When the DWDM lasers sources (supply of the DWDM system) was used, the RF uncertainty was larger than $1 \mathrm{GHz}$. It is too large for the signal transmission. Therefore, the lasers were dismounted from the board and were supplied by Agilent E3631a voltage sources. The current of the voltage source was set to $20 \mathrm{~mA}$ and it was measured by a current meter. The change of the current was $1 \%$. In that case the RF moved in a $315 \mathrm{MHz}$ wide range.

In the next step one of the voltage sources was changed to a laser driver from Thorlabs. It halved the frequency uncertainty to $147 \mathrm{MHz}$. Then the laser driver was changed to another one (manufactured by ILX) to check which current source was more precise. Using the ILX current source the RF was moved in a $135 \mathrm{MHz}$ wide spectrum range, which is less by $12 \mathrm{MHz}$ than in the previous measurement. If the lasers were driven by laser drivers the RF uncertainty was $66 \mathrm{MHz}$. The frequency of the photomixed signal can be more stable if one of the lasers is constantly supplied as the simulation showed. Therefore, one

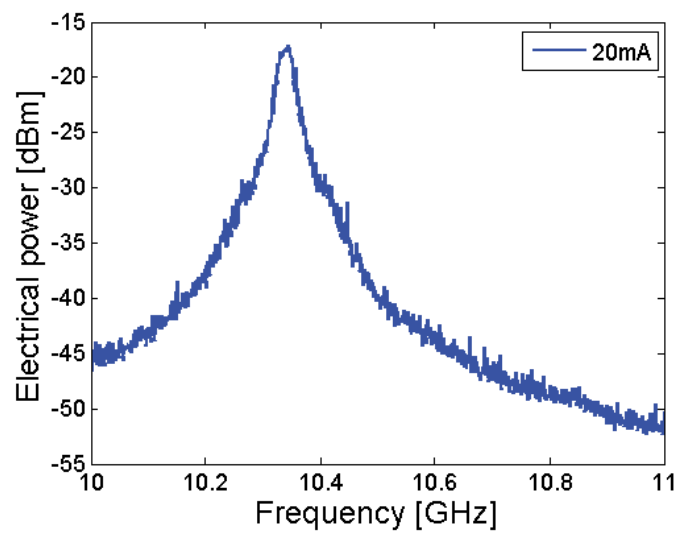

DWDM laser was exchanged to a laboratory CW laser source, which has its own driving and temperature control circuit and it cannot be manually controlled. This laser source was stable enough to decrease the RF instability to 69 or $15 \mathrm{MHz}$ depending on the laser driver. The detected spectrum can be seen in Fig. 7.

The uncertainty in the photomixed signal frequency becomes higher if the supply current of the laser is increased. The laser wavelength is dependent on the bias current and consequently varying the bias current the frequency of the mixing product is changed. Now the laser current was set to $100 \mathrm{~mA}$ and its result is that the RF can be found in a $97 \mathrm{MHz}$ wide spectrum range. Frequency of the DWDM lasers changed $1 \mathrm{GHz}$ in average if the supply current changed $1 \mathrm{~mA}$. That is the same value as in our applied laser diode model of the VPI. However, the frequency fluctuation is in the $\mathrm{MHz}$ range instead of the simulation results providing about $1 \mathrm{GHz}$. In the measurements the voltage sources generated a supply current with $1 \%$ stability. The measured $315 \mathrm{MHz}$ instability is fewer than 0.2 part of the simulated frequency shift. It can come from the differences between the DWDM laser diode and the modelled laser diode. All in all, we showed the supply current stability is very critical in this carrier generation method. It influences the linewidth caused signal distortion significantly. Higher supply current caused smaller linewidth effect on the RF could not be observed (Fig. 7), because of the supply instability.

\section{Conclusion}

We showed an RF carrier generation techniques which can be used in terahertz communications. Mixing two independent lasers suffers from many instability problems. In the simulations it is easy to generate a stable RF carrier with this method, but the reality showed a totally different result. The stability of the supply current turned to be a very critical problem of this method. If it is not stable enough, the frequency wander can reach a few GHz. The laser linewidth affects the RF signal bandwidth in a nonlinear way. It has a bottom value which cannot be decreased by a laser with smaller linewidth. We also showed that the laser linewidth caused RF spectral broadening has a saturation. However, this effect is much smaller than the

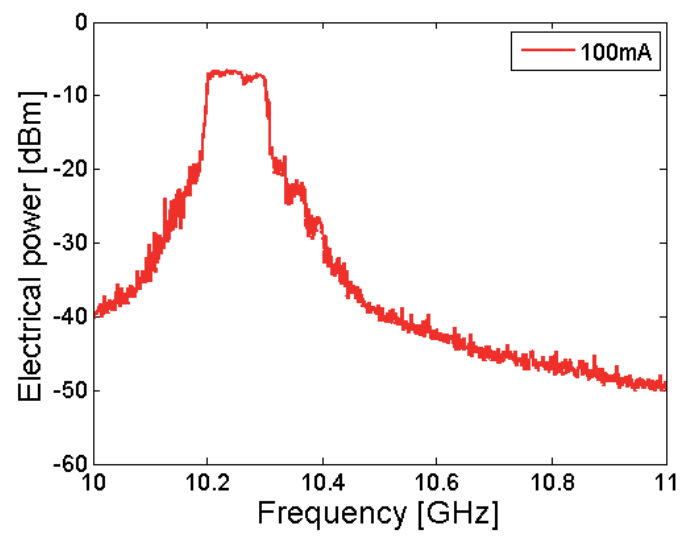

Fig. 7 Electrical spectrum of the photomixed DWDM and laboratory lasers, if the DWDM laser was driven by the ILX laser driver. 
power supply instability caused frequency uncertainty. The present signal generation method has a significant advantage i.e. the easy frequency tuning by varying the temperature of one of the lasers.

\section{Acknowledgement}

The authors would like to acknowledge the support of the Furukawa Electric Institute of Technology Ltd. (FETI Kft.) to their work.

\section{References}

[1] Kleine-Ostmann, T., Nagatsuma, T. "A Review on Terahertz Communications Research." Journal of Infrared, Millimeter, and Terahertz Waves. 32 (2). pp. 143-171. 2011. DOI: 10.1007/s10762-010-9758-1

[2] Nagatsuma, T., Song, H-J., Kado, Y. "Challenges for Ultrahigh-Speed Wireless Communications Using Terahertz Waves." Terahertz Science and Technology. 3 (2). pp. 55-65. 2010.

[3] Nagatsuma, T., Horiguchi, S., Minamikata, Y., Yoshimizu, Y., Hisatake, S., Kuwano, S., Yoshimoto, N., Terada, J., Takahashi, H. "Terahertz wireless communications based on photonics technologies." Optics Express. 21 (20). pp. 23736-23747. 2013. DOI: 10.1364/oe.21.023736

[4] Song, H-J., Nagatsuma, T. "Present and Future of Terahertz Communications." IEEE Transactions on Terahertz Science and Technology. 1 (1). pp. 256-263. 2011. DOI: 10.1109/tthz.2011.2159552

[5] Seeds, A. J., Williams, K. J. "Microwave Photonics." Journal of Lightwave Technology. 24 (12). pp. 4628-4641. 2006.

DOI: $10.1109 /$ JLT.2006.885787

[6] Berceli, T., Herczfeld, P. R. "Microwave Photonics-A Historical Perspective." IEEE Transaction on Microwave Theory and Techniques. 58 (11). pp. 2992-3000. 2010. DOI: 10.1109/tmtt.2010.2076932

[7] Agrawal, G. P. "Fiber-Optic Communication Systems." 3rd ed. John Wiley \& Sons Inc. 2002. 